\title{
Parallel Digital Watermarking Process on Ultrasound Medical Images in Multicores Environment
}

\author{
Hui Liang Khor, Siau-Chuin Liew, and Jasni Mohd. Zain \\ Faculty of Computer Systems and Software Engineering, University Malaysia Pahang, Lebuhraya Tun Razak, Gambang, \\ 26300 Kuantan, Pahang Darul Makmur, Malaysia
}

Correspondence should be addressed to Hui Liang Khor; hlkhorr@yahoo.com

Received 20 November 2015; Revised 2 January 2016; Accepted 4 January 2016

Academic Editor: Shuihua Wang

Copyright (c) 2016 Hui Liang Khor et al. This is an open access article distributed under the Creative Commons Attribution License, which permits unrestricted use, distribution, and reproduction in any medium, provided the original work is properly cited.

With the advancement of technology in communication network, it facilitated digital medical images transmitted to healthcare professionals via internal network or public network (e.g., Internet), but it also exposes the transmitted digital medical images to the security threats, such as images tampering or inserting false data in the images, which may cause an inaccurate diagnosis and treatment. Medical image distortion is not to be tolerated for diagnosis purposes; thus a digital watermarking on medical image is introduced. So far most of the watermarking research has been done on single frame medical image which is impractical in the real environment. In this paper, a digital watermarking on multiframes medical images is proposed. In order to speed up multiframes watermarking processing time, a parallel watermarking processing on medical images processing by utilizing multicores technology is introduced. An experiment result has shown that elapsed time on parallel watermarking processing is much shorter than sequential watermarking processing.

\section{Introduction}

The technological advancement in communication network has facilitated healthcare professionals across the world in accessing electronic medical records, such as medical images, and obtaining second opinions for high-quality diagnosis. As a consequence, medical images are exposed to security threat such as tampering of images, which may lead to wrong diagnosis and treatment [1]; thus a digital watermarking on medical image is introduced. So far most of the watermarking research has been done on single frame medical image which is impractical in the real environment, where most of the ultrasound medical images consist of multiframes; thus a digital watermarking on multiframes medical images is proposed. Watermarking could be applied on ultrasound medical images frame by frame sequentially but it would be time consuming for a large dataset; thus a parallel environment is necessary for speeding up multiframes watermarking process by utilizing multicore technology. The performance improvements gained by the use of a multicore processor largely depended on the software algorithms used and their implementation. According to Amdahl's law, the possible gains are only limited on software portion that can be run in parallel simultaneously on multiple cores. Our work explores efficient parallel implementations of the digital watermarking scheme in multicore environment.

\section{Theory: Concept of Digital Watermarking}

Digital watermarking is the technology that imperceptibly modifies original data and embeds them directly into image. Digital watermarking in general is comprised of three major components [2]:

(1) Watermark generator: a desired watermark(s) is generated for particular applications, which are optionally dependent on some keys.

(2) Watermark embedder: watermark(s) are embedded into the object, sometimes based on an embedding key.

(3) Watermark detector: detecting the existence of some predefined watermark in the object. It is sometimes desirable to extract a message as well. 
TABLE 1: Comparison between task parallelism and data parallelism.

\begin{tabular}{ll}
\hline Task parallelism & Data parallelism \\
\hline $\begin{array}{l}\text { It is a form of parallelization of computer code across multiple } \\
\text { processors in parallel computing environments [22]. }\end{array}$ & $\begin{array}{l}\text { It is a form of parallelization of computing across multiple } \\
\text { processors in parallel computing environments [23]. }\end{array}$ \\
\hline $\begin{array}{l}\text { Task parallelism focuses on distributing execution processes } \\
\text { (threads) across different parallel computing nodes [23]. }\end{array}$ & $\begin{array}{l}\text { Data parallelism focuses on distributing the data across different } \\
\text { parallel computing nodes. }\end{array}$ \\
\hline
\end{tabular}

The purpose of medical image security is to maintain privacy of the patient information in the image and to assure data integrity that prevents the image from tampering [3]. Watermarking can be used in medical images to prevent unauthorized modification by authenticating the content of the image. Tamper localization capable watermarking scheme can detect and locate modification of pixel values on the image [4]. The tampered area can be recovered by retrieving the original pixel values that were stored on the image itself as a watermark. Tamper localization is useful for deducing the motive of the tampering and whether any modification is legitimate [5]. Liew and Zain proposed a reversible watermarking scheme (TALLOR watermarking scheme) by dividing image into ROI (Region of Interest) and RONI (Region of Noninterest) [6]. ROI is the significant part of the medical images that is used by doctors to diagnose the patient, and RONI is the area outside the ROI. Watermarking for tamper detection and recovery is done in the ROI area based on Jasni's scheme [7]. The original Least Significant Digits (LSBs) that are removed in watermark embedding process are stored in RONI after compression. The stored LSBs later can be used to restore the image to its original bits value so the watermarking scheme can be reversible [8]. The research was conducted on a single frame of ultrasound which is impractical in the real world; thus a digital watermarking on multiframes medical images is proposed. In order to speed up multiframes watermarking processing time, a parallel computation in multicores watermarking processing on medical images is introduced.

\section{Theory: Parallel Computing in Multicores}

In recent years there has been a surge of interest in running application in parallel to take advantage of multiprocessor and multicore systems. Developments in microprocessor technologies have resulted in most processors having multiple computing cores in a single chip [9]. Parallel computing is a concept of performing tasks simultaneously by partitioning a large and complex problem into smaller tasks and solving each of them concurrently. There are two forms of parallelism: task parallelism and data parallelism (as shown in Table 1).

Data parallelism emphasizes the distributed (parallelized) nature of the data, as opposed to the processing (task parallelism) [10]. Data parallelism is adopted in this experiment since each processor performs the same code (watermarking code) on different pieces of distributed ultrasound frames.

Optimal speedup from parallelization should be linear if the number of processing elements is inversely proportional to its run time. However, not many parallel algorithms achieve optimal speedup. Most of them have a near-linear speedup for small numbers of processing elements, which flattens out into a constant value for large numbers of processing elements [11].

According to Amdahl's law, the overall speedup from parallelization would be restricted by a small portion of the program which cannot be parallelized. A large and complex program usually consists of several parallelizable parts and several nonparallelizable (sequential) parts. If $\alpha$ is the fraction of running time a program spends on nonparallelizable parts [12], then

$$
\lim _{P \rightarrow \infty} \frac{1}{(1-\alpha) / P+\alpha}=\frac{1}{\alpha} .
$$

It is the maximum speedup with parallelization of the program, with $P$ being the number of processors used. If the sequential portion of a program accounts for $10 \%$ of the runtime $(\alpha=0.1)$, then a 10x speedup will be the maximum, regardless of how many processors are added. This generates an upper limit on the usefulness of adding more parallel execution units [13].

Gustafson's law is another law in computing, closely related to Amdahl's law [9]. It states that the speedup with $P$ processors is

$$
S(P)=P-\alpha(P-1)=\alpha+P(1-\alpha) .
$$

Amdahl's law assumes that the total amount of work to be done in parallel is also independent of the number of processors, whereas Gustafson's law assumes that the total amount of work to be done in parallel varies linearly with the number of processors [12].

Applications are often classified based on the frequency of synchronization and communication needs between their subtasks. Fine-grained parallelism is where an application has a high rate of communication among subtasks; coarsegrained parallelism is where an application does not communicate many times per second, and it is embarrassingly parallel if an application seldom or never has to communicate. Embarrassingly parallel applications are considered the easiest to parallelize [14]. In the embarrassingly parallel problems, speedup factors could be achieved near the number of cores, or even more if the problem is partitioned enough to fit within each core's cache(s), avoiding use of much slower main system memory.

\section{Parallel Computing with MATLAB}

4.1. How Parallel Computing Runs a Job. The MATLAB job scheduler (MJS) is the process that coordinates the jobs 


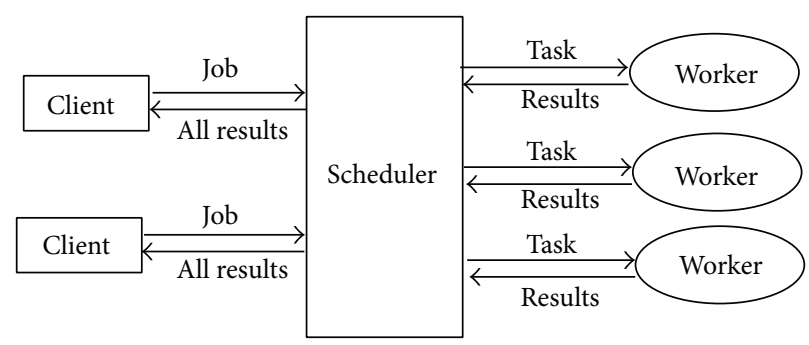

FIgURE 1: Interactions of Parallel Computing Session.

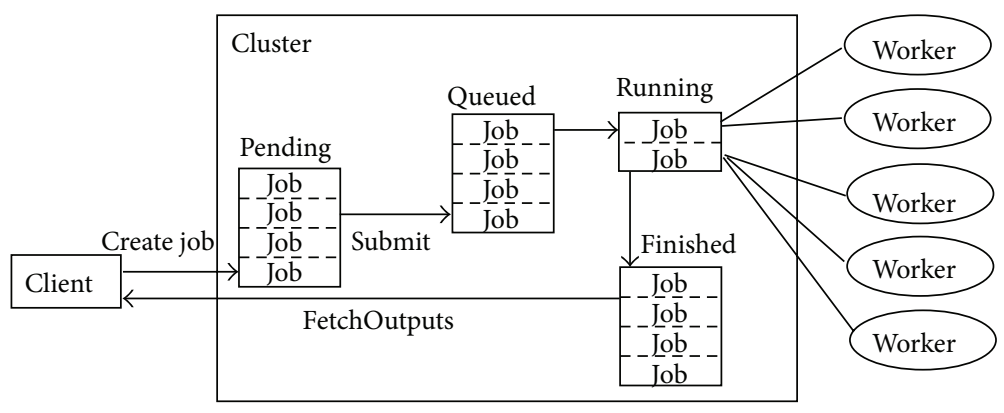

FIGURE 2: Stage of a job.

execution and their tasks evaluation. The MJS can be run on any machine on the network. The MJS runs the submitted jobs in queue order, unless any jobs in its queue are promoted, demoted, cancelled, or deleted. MJS assigns task from the running job to each worker for execution and fetches result from workers upon the task completion. The cycle is repeated with another task. When all tasks for a running job have been assigned to workers, the MJS starts running the next job on the next available worker. Tasks were executed simultaneously by all workers in order to speed up execution of large MATLAB jobs. The MJS then returns the results of all the tasks in the job to the client session (as shown in Figure 1) [15]. In this research, client and MJS are located in a single computer where a client (end user) has sent a job (a stack of ultrasound medical images) into MJS where it segregates the ultrasound medical images into multiple tasks (subset of ultrasound medical images frames) into workers (cores) and executes watermarking process in parallel. For example, in quad cores computer, MJS will segregate 15 frames of ultrasound medical images into 4,4,4,3 frames to each core/worker, respectively. The details process will be discussed in Section 5.

4.2. Life Cycle of a Job. Job progresses through a number of stages upon its creation. In the MJS (or other schedulers), each stage of a job is categorized by their state, such as pending, queued, running, or finished (refer to Table 2). Figure 2 illustrates the stages in the life cycle of a job. Functions used in job management are createJob, submit, and fetchOutputs [14].

Table 2 describes each stage in the life cycle of a job [15].

\section{Research Methodology: Sequential and Parallel Watermarking Embedding and Authentication Process}

In watermarking process on a single frame of ultrasound medical image, watermark is embedded into ultrasound medical images and becomes an input file for watermarking authentication process (as shown in Figure 3). The purpose of authentication process is to localize and recover the tamper region in medical images. In other words, the prerequisite of watermarking authentication process is watermarked ultrasound medical images, in which it is the output file generated by watermarking embedding process. Process watermarking frame by frame sequentially was time consuming; thus parallel computing on multicores was introduced to solve the problem concern. This is accomplished by dividing ultrasounds frames into tasks to each core, respectively, and performing watermarking process simultaneously with others.

Two modes of watermarking process in multiframes will be developed and compared; there is sequential (by using for loop) versus parallel watermarking process (watermarking on multicores); the purpose is to prove that the parallel watermarking will have a significant improvement on elapsed time. In both sequential watermarking embedding and authentication process, watermarking is processed frame by frame sequentially by using a control loop. Therefore the elapsed time is proportional to the number of frames processed. The elapsed time could be reduced by using parallel computing on multicore processing technique and this technique enables ultrasound frames to be divided and distributed to multicore for parallel watermarking processing. 
TABLE 2: Stage in life cycle of a job.

\begin{tabular}{|c|c|c|c|}
\hline Job stage & \multicolumn{3}{|l|}{ Description } \\
\hline Pending & \multicolumn{3}{|c|}{$\begin{array}{l}\text { A job is created on the scheduler with the createJob function in client session of Par } \\
\text { software. The job's first state is pending. This is when the job was defined by adding }\end{array}$} \\
\hline Queued & \multicolumn{3}{|c|}{$\begin{array}{l}\text { When the submit function is executed on a job, the MJS or scheduler places the job } \\
\text { state is queued. The scheduler executes jobs in the queue in the sequence in which th } \\
\text { moving up the queue as the jobs before them are finished. The sequence of the jobs } \\
\text { with the promote and demote functions. }\end{array}$} \\
\hline Running & \multicolumn{3}{|c|}{$\begin{array}{l}\text { When a job reaches the top of the queue, the scheduler distributes the job's tasks to } \\
\text { evaluation. The job's state is now running. If more workers are available than are requ } \\
\text { scheduler begins executing the next job. In this way, there can be more than one job }\end{array}$} \\
\hline Finished & \multicolumn{3}{|c|}{$\begin{array}{l}\text { When all of a job's tasks have been evaluated, the job is moved to the finished state. } \\
\text { be retrieved from all the tasks in the job with the function fetchOutputs. }\end{array}$} \\
\hline Failed & \multicolumn{3}{|c|}{$\begin{array}{l}\text { When using a third-party scheduler, a job might fail if the scheduler encounters an } \\
\text { execute its commands or access necessary files. }\end{array}$} \\
\hline Deleted & \multicolumn{3}{|c|}{$\begin{array}{l}\text { When a job's data has been removed from its data location or from the MJS with th } \\
\text { the job in the client is deleted. This state is available only as long as the job object re }\end{array}$} \\
\hline & $\begin{array}{l}\text { Input ultrasound } \\
\text { medical images }\end{array}$ & $\begin{array}{l}\text { Watermarking } \\
\text { embedding process }\end{array}$ & $\begin{array}{l}\text { Generate } \\
\text { watermarked } \\
\text { ultrasound medical } \\
\text { images }\end{array}$ \\
\hline & $\begin{array}{l}\text { Generate tampering } \\
\text { message and a } \\
\text { recovered DICOM } \\
\text { file }\end{array}$ & $\begin{array}{l}\text { Watermarking } \\
\text { authentication } \\
\text { process }\end{array}$ & $\begin{array}{l}\text { Tampering on } \\
\text { watermarked } \\
\text { ultrasound medical } \\
\text { images }\end{array}$ \\
\hline
\end{tabular}

FIGURE 3: Watermarking process flow on a single frame of ultrasound medical image.

5.1. Sequential Watermarking Process. Sequential watermarking embedding and authentication process are sharing a common framework, where ultrasound medical images in DICOM format are read and perform watermarking process frame by frame sequentially by using a for loop. Processed frames will then concatenate into a variable named as " $\mathrm{A}$ " which will convert into DICOM format at the end of the watermarking process. The relationship between both processes is that the output file of watermarking embedding process is the input file for watermarking authentication process. The difference between them is that authentication process has an additional step in identifying the tampered frames; if it is 0 , it means nontampered; else it is tampered and then image recovery is performed and tampered frame number is recorded and will be displayed upon the completion of watermarking process (as demonstrated in Figure 4). The main algorithms of parallel watermarking process are dividing volumetric ultrasound medical images and distributing them into a number of cores and executing sequential watermarking processes on each core in parallel; therefore a successful sequential watermarking process is a prerequisite in parallel watermarking process. The details of parallel watermarking process will be discussed in Section 5.2.
5.2. Parallel Watermarking Process. In parallel watermarking process (as illustrated in Figures 5 and 6), ultrasound multiframes medical images were loaded into a quad core microprocessor/cluster and create a job on the scheduler; the job is then divided into tasks according to the number of cores in the microprocessor.

The code implemented enables cluster to autodetect the number of cores available in the processor; if the processor used is a quad core, then the job is divided into 4 tasks, where ultrasound frames are equally divided by 4 ; for example, if the total number of ultrasound frames is 30 , then it will be divided into 8,8,7,7 frames, and if the total number of frames is 15 , then it will divided into $4,4,4,3$ frames. Those divided frames will then distribute to 4 cores, respectively. In each core, watermarking process is carried out sequentially on the divided frames and at the same time it runs concurrently with other cores (as illustrated in Figure 7).

It is important to ensure that the generated frames output is in order after parallel watermarking process; therefore the frame number has to be assigned and keep track before the frames are sent to the cores, respectively. Upon the tasks completion, tasks were reassembled into a job, whereby all the frames will concatenate into an array and submit back to the cluster. The result is retrieved from all the tasks in 


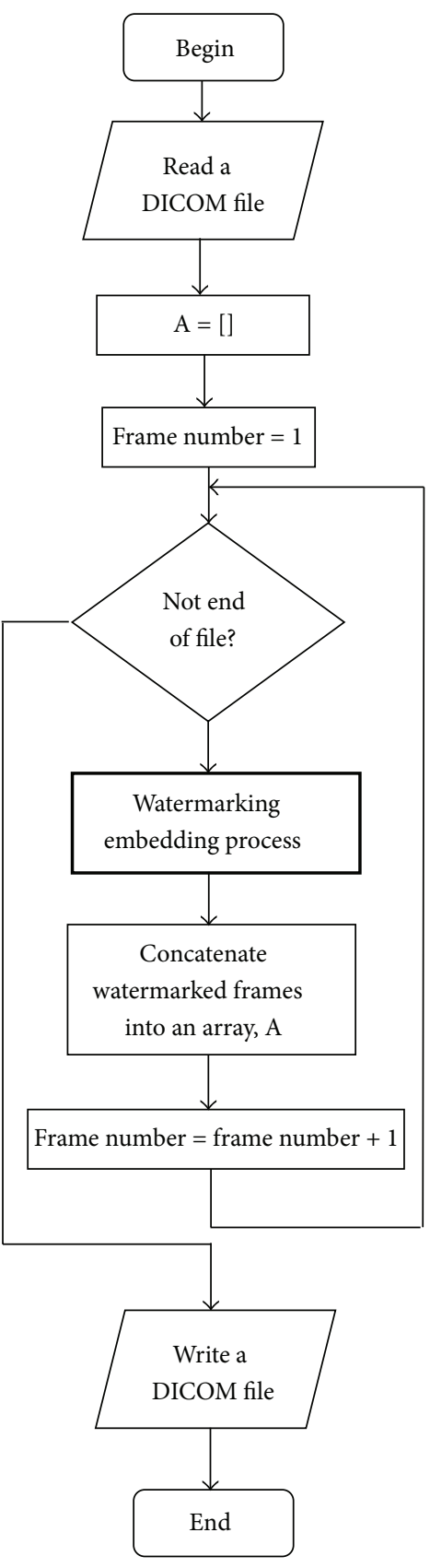

(a)

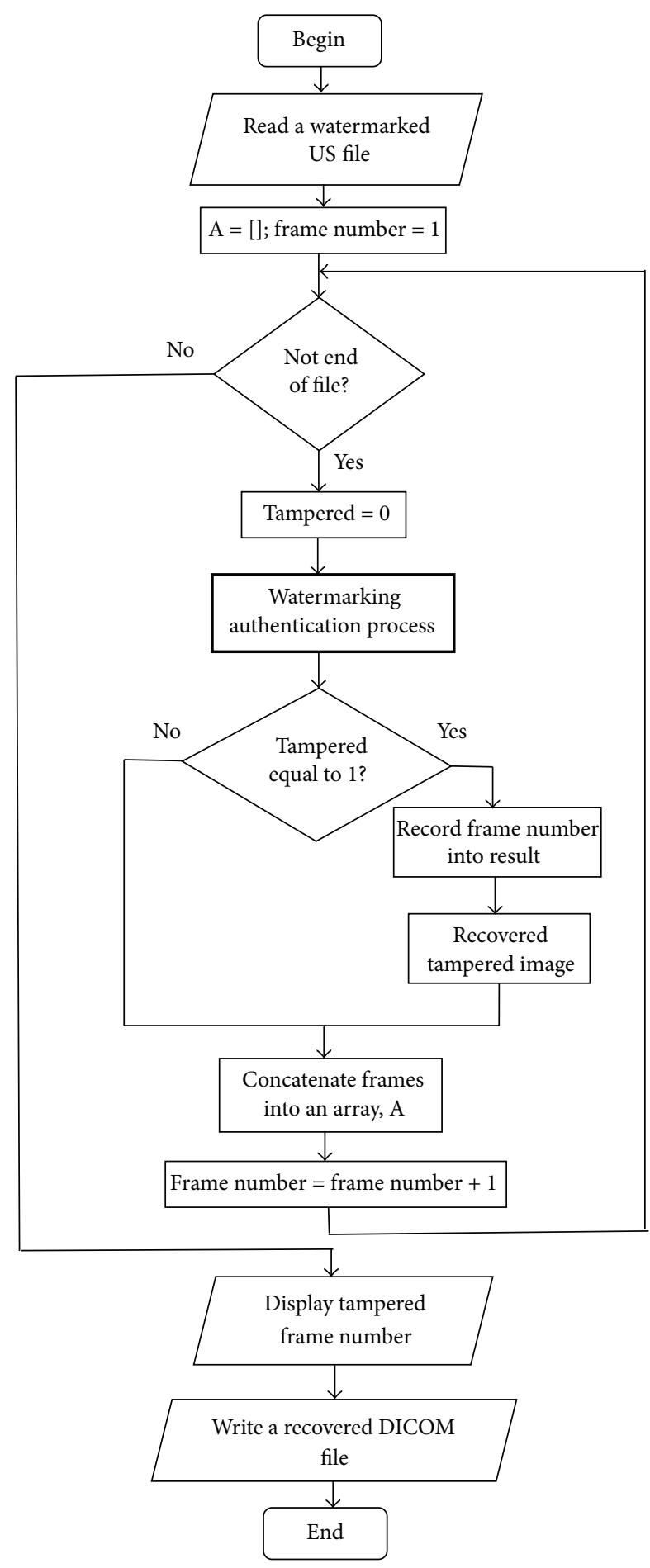

(b)

FIGURE 4: Program flow chart for (a) sequential watermarking embedding process and (b) sequential watermarking authentication process.

the job with the function fetch output. All the frames will concatenate according to the frame number order and write into a DICOM file. A job is deleted on two circumstances:

(1) When the scheduler encounters an error.

(2) When the job is finished.
Both parallel watermarking embedding and authentication process have a similar process flow as described above, except the process applied on each task, input and output files as listed in Table 3. The input file of authentication process is the tampered output file of embedding process. 


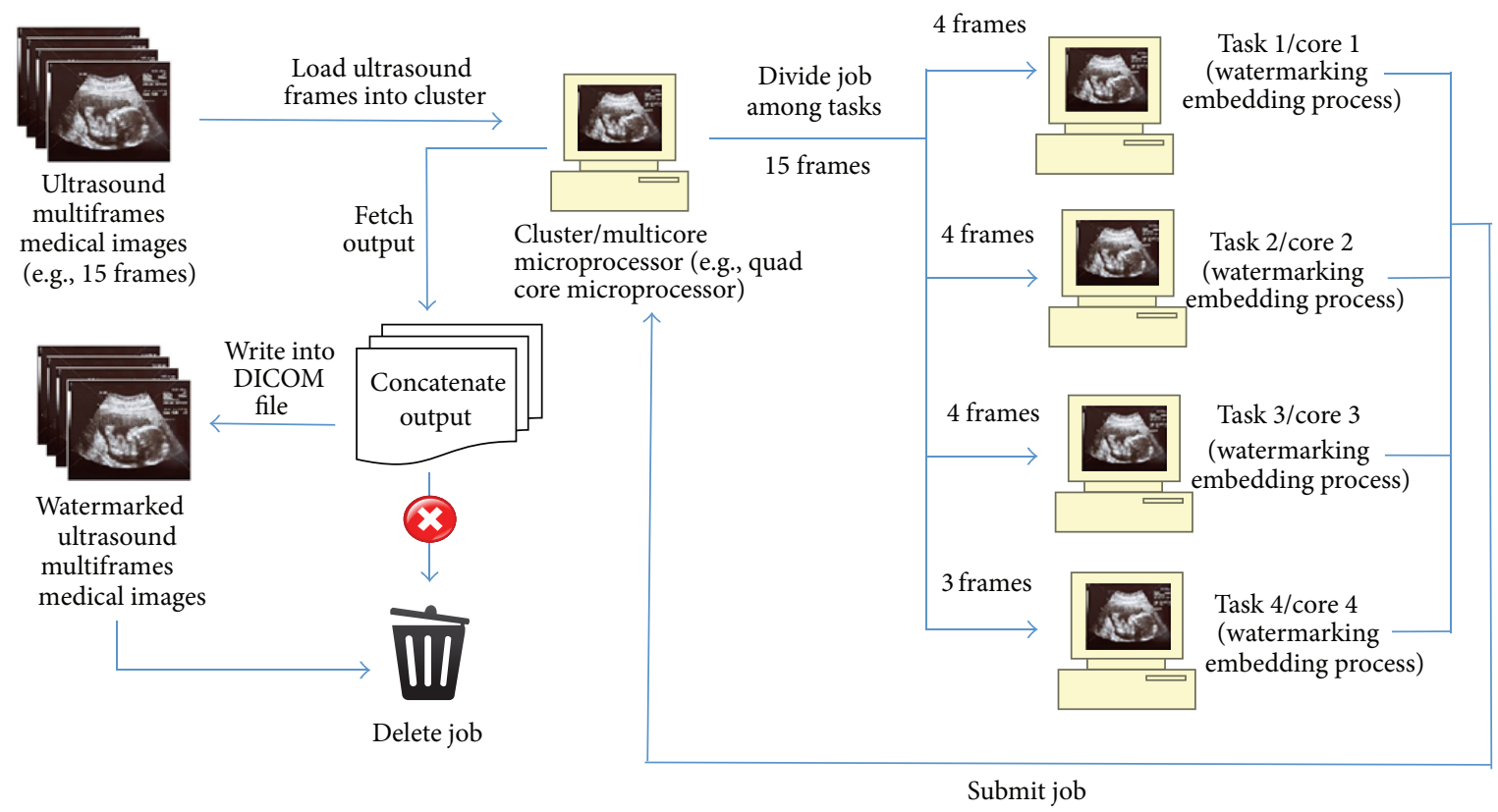

Figure 5: Parallel watermarking embedding process.

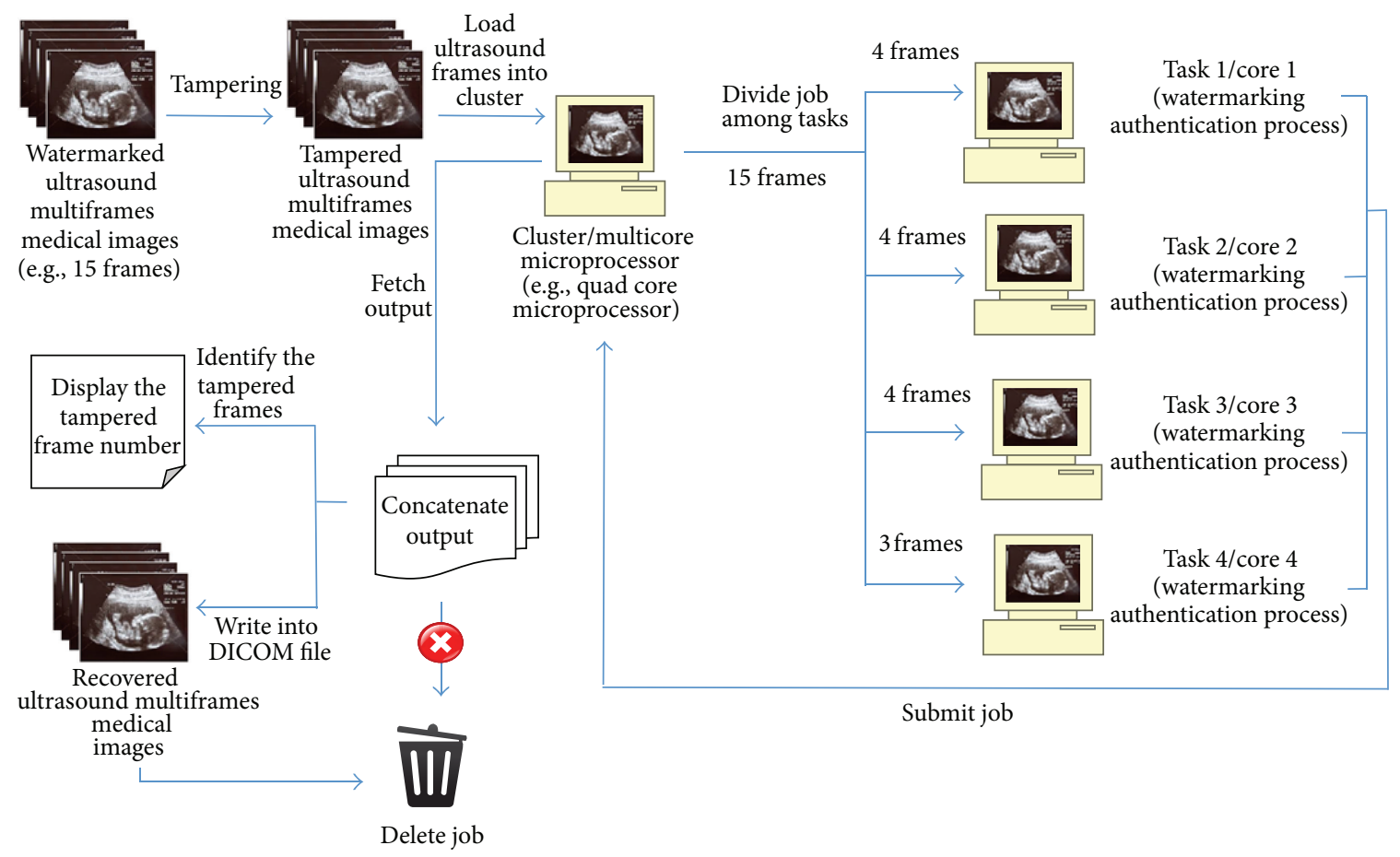

FIGURE 6: Parallel watermarking authentication process.

\section{Experimental Design and Set-Up}

TALLOR watermarking scheme, developed by Liew and Zain [4], will be executed in sequential and parallel modes. The elapsed time obtained from both modes will be compared and speedup factor of parallel relative to sequential watermarking process will be measured. It is to verify the efficiency of parallel framework.

Three important performance metrics were studied. These are 
TABLE 3: The relationship between parallel watermarking embedding and authentication process.

\begin{tabular}{|c|c|c|c|}
\hline Parallel watermarking & Input file & $\begin{array}{l}\text { Process applied on } \\
\text { each task }\end{array}$ & Output file \\
\hline Embedding process & $\begin{array}{l}\text { Ultrasound (US) } \\
\text { multiframe medical } \\
\text { images/raw file }\end{array}$ & $\begin{array}{l}\text { Watermarking } \\
\text { embedding process }\end{array}$ & $\begin{array}{c}\text { Watermarked US } \\
\text { multiframe medical } \\
\text { images }\end{array}$ \\
\hline $\begin{array}{l}\text { Authentication } \\
\text { process }\end{array}$ & $\begin{array}{c}\text { Tampered } \\
\text { watermarked US } \\
\text { multiframe medical } \\
\text { images }\end{array}$ & $\begin{array}{l}\text { Watermarking } \\
\text { authentication } \\
\text { process }\end{array}$ & $\begin{array}{c}\text { Recovered US } \\
\text { medical images and a } \\
\text { message of tampered } \\
\text { frame numbers }\end{array}$ \\
\hline
\end{tabular}

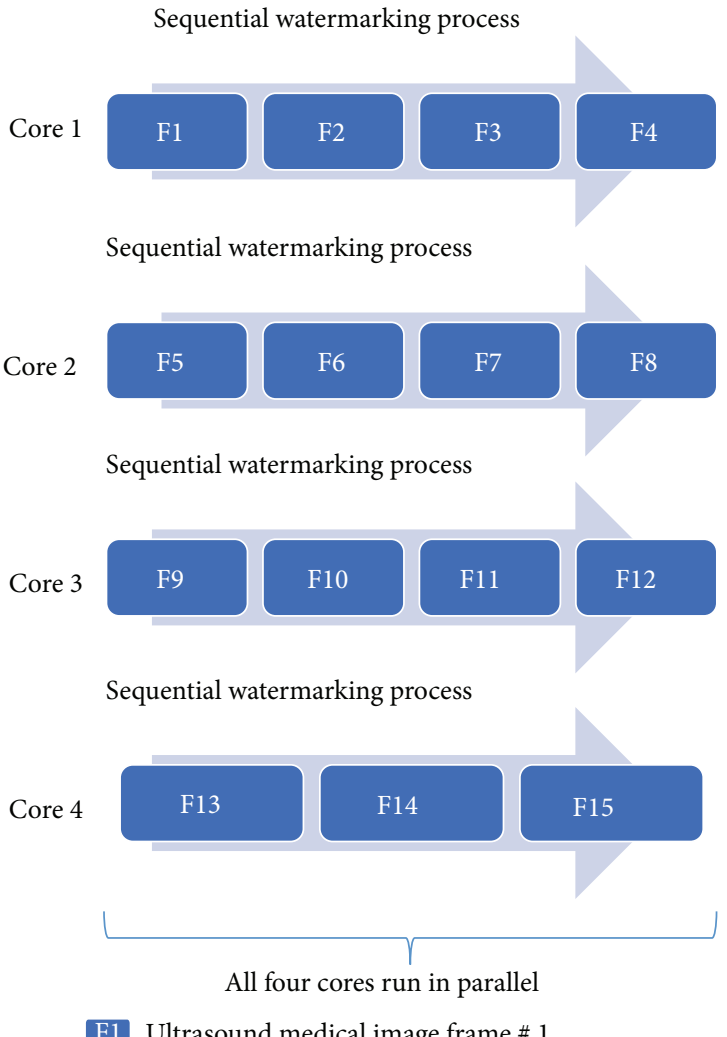

F1 Ultrasound medical image frame \# 1

FIGURE 7: Inside look of parallel watermarking process that runs on 15 frames of ultrasound medical images.

(1) imperceptibility: testing the quality of medical images in terms of invisibility of watermarking in multiframes environment;

(2) elapsed time: the time taken to perform watermarking embedding and authentication process on medical images in multiframes environment;

(3) robustness to tampering: testing the effectiveness and efficiency of the tamper detection, localization, and recovery function in multiframes environment.

The evaluation was performed by running MATLAB (The Mathworks, Inc., Natick, MA, USA) program on a laptop with quad core CPU of Intel ${ }^{\circ}$ Core $^{\mathrm{rw}}$ i7-3630QM CPU @ 2.4 GHz, $2401 \mathrm{MHz}, 4$ Core(s), 8 Logical Processor(s), and RAM of
8 GB. Three samples of ultrasound medical images in DICOM format were used to test the system (as shown in Table 4).

\section{Experimental Result}

7.1. Imperceptibility. The perceptibility of a watermarked image can be judged according to its fidelity and quality. Fidelity measures the similarity between images before and after watermarking [16]. A high fidelity means that watermarked image is very similar to the original image. The meansquared-error (MSE) and peak signal-to-noise ratio (PSNR) were calculated by comparing the watermarked image and original image. Watermarked images may bear visible or invisible distortion due to the embedding process. One way to quantify distortion is the mean-square error. If $\mathbf{I}_{i}^{\prime}$ is a vector of $n$ predictions, and $\mathbf{I}_{i}$ is the vector of observed values corresponding to the inputs to the function which generated the predictions, then the MSE of the predictor can be estimated by

$$
\operatorname{MSE}=\frac{1}{n} \sum_{i}^{n}\left(\mathbf{I}_{i}^{\prime}-\mathbf{I}_{i}\right)^{2}
$$

MSE is the average term by term difference between the original image, $\mathbf{I}$, and the watermarked image, $\mathbf{I}^{\prime}$. If $\mathbf{I}$ and $\mathbf{I}^{\prime}$ are identical, then $\operatorname{MSE}\left(\mathbf{I}^{\prime}, \mathbf{I}\right)=0$. A related distortion measure is the peak signal-to-noise ratio (PSNR), measured in decibels $(\mathrm{dB})$. The problem with mean-square error is that it depends strongly on the image intensity scaling while PSNR rectifies this problem by scaling the mean-square error according to the image range [17]. PSNR is defined as follows:

$$
\operatorname{PSNR}(\mathrm{dB})=10 \log _{10} \frac{\max \mathrm{I}^{2}}{\mathrm{MSE}^{\prime}},
$$

where max I is the peak value of the original image. If the signals are identical, then PSNR is equal to infinity. A high PSNR represents a high fidelity of a watermarked image. In this thesis, PSNR is used as a measurement for image fidelity. A high-quality watermarked image does not have any obvious noticeable distortion caused by the watermark embedding process. The assessment of quality is usually evaluated by human observers and is influenced by personal preferences which are subjective in nature.

Three different sets of ultrasound medical images which contain thirty and fifteen frames have been watermarked 
TABLE 4: Ultrasound medical images samples properties.

\begin{tabular}{lccc}
\hline Ultrasound medical images & Image dimension in pixels & Bits per pixel & Number of frames \\
\hline Ultraound_Sample_1.dcm & $640 \times 480$ & 8 & 30 \\
Ultraound_Sample_2.dcm & $640 \times 480$ & 8 & 15 \\
Ultraound_Sample_3.dcm & $640 \times 476$ & 8 & 15 \\
\hline
\end{tabular}

in two different ways: (1) sequentially and (2) parallel. It is important to ensure that the quality and fidelity of images were not affected by the way watermarking embedding process performed. Both sequential and parallel watermarking embedding processes have produced the same MSE and PSNR result for each frame as indicated in Table 5 except some negligible differences in the highlighted areas. This means that the operation either in sequential or in parallel mode does not affect the image quality and its fidelity. The PSNR values reflect the medical image integrity and high PSNR values indicate lesser distortion on medical image after watermarking process. PSNR value reflected medical image fidelity; ideally the watermarked medical image should be visually indistinguishable as original image. The PSNR values are calculated for all images ranging within $48.29 \sim 48.74 \mathrm{~dB}$, which are within the acceptable range for diagnosis purposes and it has achieved imperceptibility as shown in Figure 9 where the images before and after watermarking embedding process are visually indistinguishable as the original images.

7.2. Elapsed Time. Elapsed time is the time taken to perform watermarking embedding (Figure 8) and authentication process (Figure 12) on medical images in multiframes environment. This section is to test the speedup factor in parallel mode as compared to sequential mode in watermarking process. The formula of speedup factor is defined as follows:

$$
\text { Speed up }=\frac{\text { Elapsed time in sequnetial mode }}{\text { Elapsed time in parallel mode }} \text {. }
$$

Speedup factor is to measure the speed of parallel mode by factors relative to sequential mode. For example, if the speedup is 3, this means parallel process is three times faster than sequential mode.

7.2.1. Watermarking Embedding Process. Table 6 shows that watermarking embedding process in parallel has achieved a significant speedup $(14.13 \sim 19.29)$ relative to sequential process. In Figure 10, the elapsed time in sequential watermarking embedding process on sample 22 and sample_3 is similar but increases by double in sample_1; this means that the elapsed time in sequential process is proportional to the number of frames, whereas in parallel watermarking embedding process, the elapsed time results are consistent despite the number of frames processed. In conclusion, the number of frames does not have much impact on the parallel process as compared to sequential process. The watermarking embedding scheme is pixel oriented, which means different ultrasound sample with the same frame size will produce a similar result.

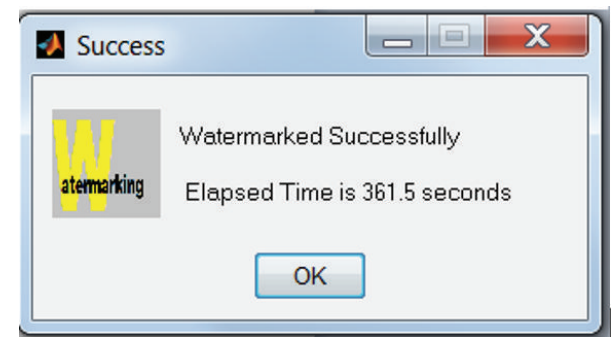

FIgURE 8: Message displayed upon the completion of sequential watermarking embedding process.

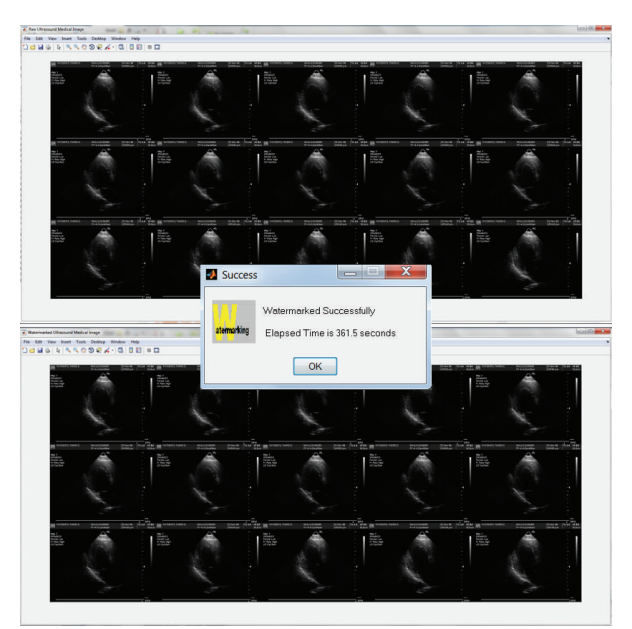

FIgURE 9: The output of raw and watermarked ultrasound medical images after a sequential watermarking embedding process.

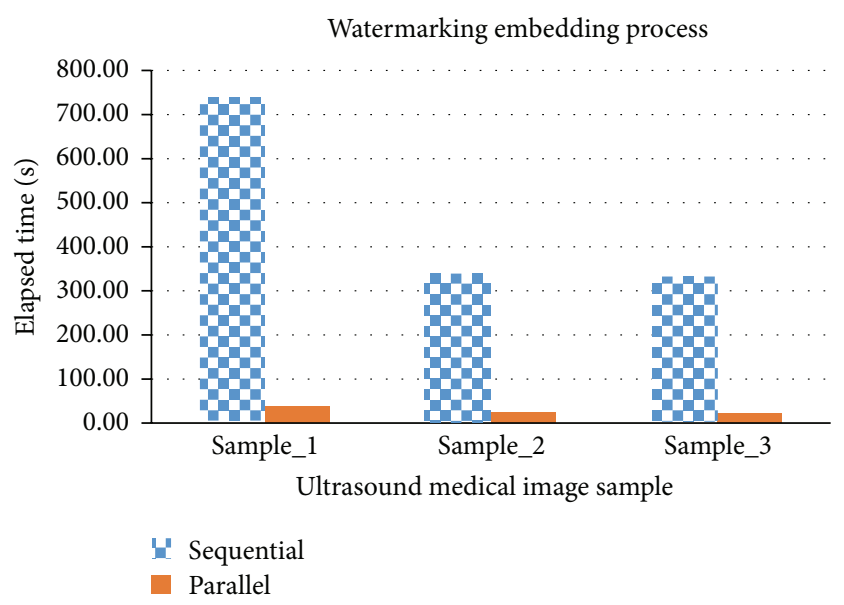

FIGURE 10: Sequential versus parallel watermarking embedding process in elapsed time. 
TABLE 5: PSNR and MSE values for three sets of watermarked ultrasound images produced by watermarking embedding process in different modes: sequential and parallel modes.

(a) Watermarking embedding process on Ultrasound_Sample_1.dcm

\begin{tabular}{|c|c|c|c|c|}
\hline \multirow{2}{*}{ Ultrasound frame number } & \multicolumn{2}{|c|}{ Sequential } & \multicolumn{2}{|c|}{ Parallel } \\
\hline & MSE & PSNR & MSE & PSNR \\
\hline 1 & 1.0444 & 47.942 & 1.0444 & 47.942 \\
\hline 2 & 1.0119 & 48.079 & 1.0119 & 48.079 \\
\hline 3 & 1.0241 & 48.027 & 1.0241 & 48.027 \\
\hline 4 & 1.0200 & 48.045 & 1.0200 & 48.045 \\
\hline 5 & 0.9827 & 48.207 & 0.9827 & 48.207 \\
\hline 6 & 1.0055 & 48.107 & 1.0055 & 48.107 \\
\hline 7 & 1.0240 & 48.028 & 1.0240 & 48.028 \\
\hline 8 & 0.99058 & 18.172 & 0.99058 & 18.172 \\
\hline 9 & 0.99704 & 48.144 & 0.99704 & 48.144 \\
\hline 10 & 1.0733 & 47.824 & 1.0733 & 47.824 \\
\hline 11 & 1.0224 & 48.035 & 1.0178 & 48.054 \\
\hline 12 & 1.0218 & 48.037 & 1.0218 & 48.037 \\
\hline 13 & 1.0732 & 47.824 & 1.0732 & 47.824 \\
\hline 14 & 1.0224 & 48.035 & 1.0224 & 48.035 \\
\hline 15 & 1.0216 & 48.038 & 1.0216 & 48.038 \\
\hline 16 & 1.0796 & 47.798 & 1.0796 & 47.798 \\
\hline 17 & 1.0237 & 48.029 & 1.0237 & 48.029 \\
\hline 18 & 1.0051 & 48.109 & 1.0051 & 48.109 \\
\hline 19 & 1.0175 & 48.055 & 1.0393 & 47.963 \\
\hline 20 & 0.97341 & 48.248 & 0.97341 & 48.248 \\
\hline 21 & 0.99134 & 48.169 & 0.99134 & 48.169 \\
\hline 22 & 1.0067 & 48.102 & 1.0067 & 48.102 \\
\hline 23 & 0.97568 & 48.238 & 0.97568 & 48.238 \\
\hline 24 & 0.99032 & 48.173 & 0.99032 & 48.173 \\
\hline 25 & 1.0008 & 48.127 & 1.0008 & 48.127 \\
\hline 26 & 0.97283 & 48.25 & 0.97283 & 48.25 \\
\hline 27 & 0.98178 & 48.211 & 0.98178 & 48.211 \\
\hline 28 & 1.0029 & 48.118 & 1.0029 & 48.118 \\
\hline 29 & 0.97323 & 48.249 & 0.97323 & 48.249 \\
\hline 30 & 0.99299 & 48.161 & 0.99299 & 48.161 \\
\hline
\end{tabular}

(b) Watermarking embedding process on Ultrasound_Sample_2.dcm

\begin{tabular}{lcccc}
\hline Ultrasound frame number & Sequential & & \multicolumn{2}{c}{ Parallel } \\
\hline 1 & MSE & PSNR & MSE & 4.0338 \\
2 & 1.0338 & 47.986 & 1.0329 & 47.986 \\
3 & 1.0329 & 47.99 & 1.0491 & 47.923 \\
4 & 1.0491 & 47.923 & 1.0506 & 47.916 \\
5 & 1.0506 & 47.916 & 1.0597 & 47.879 \\
6 & 1.0465 & 47.934 & 1.0616 & 47.81 \\
7 & 1.0616 & 47.81 & 1.0502 & 47.918 \\
8 & 1.0502 & 47.918 & 1.0458 & 47.936 \\
9 & 1.0458 & 47.936 & 1.0595 & 47.88 \\
10 & 1.0621 & 47.869 & 1.0632 & 47.865 \\
11 & 1.0632 & 47.865 & 1.0611 & 47.873 \\
12 & 1.0611 & 47.873 & 0.93946 & 48.402 \\
\hline
\end{tabular}


(b) Continued.

\begin{tabular}{llcccc}
\hline \multirow{2}{*}{ Ultrasound frame number } & \multicolumn{2}{c}{ Sequential } & & Parallel & PSNR \\
\hline 14 & MSE & PSNR & MSE & 47.898 \\
15 & 1.055 & 47.898 & 1.055 & 48.402 \\
\hline
\end{tabular}

(c) Watermarking embedding process on Ultrasound_Sample_3.dcm

\begin{tabular}{lcccc}
\hline Ultrasound frame number & Sequential & & & Parallel \\
& MSE & PSNR & MSE & 48.74 \\
\hline 1 & 0.86921 & 48.74 & 0.86921 & 48.7 \\
2 & 0.87713 & 48.7 & 0.87713 & 48.668 \\
3 & 0.88358 & 48.668 & 0.88358 & 48.587 \\
4 & 0.9003 & 48.587 & 0.9003 & 48.575 \\
5 & 0.90273 & 48.575 & 0.90273 & 48.494 \\
6 & 0.91967 & 48.494 & 0.91967 & 48.531 \\
7 & 0.91198 & 48.531 & 0.91198 & 48.537 \\
8 & 0.9108 & 48.537 & 0.9108 & 48.523 \\
9 & 0.91355 & 48.523 & 0.91355 & 48.434 \\
10 & 0.93258 & 48.434 & 0.93258 & 48.332 \\
11 & 0.9548 & 48.332 & 0.9548 & 48.34 \\
12 & 0.95307 & 48.34 & 0.95307 & 48.297 \\
13 & 0.96239 & 48.297 & 0.96239 & 48.406 \\
14 & 0.93856 & 48.406 & 0.93856 & 48.459 \\
\hline
\end{tabular}

TABLE 6: Sequential versus parallel watermarking embedding process in elapsed time.

\begin{tabular}{|c|c|c|c|c|c|}
\hline \multirow{2}{*}{$\begin{array}{l}\text { Input file } \\
\text { (ultrasound medical } \\
\text { images) }\end{array}$} & \multirow[t]{2}{*}{ Number of frames } & \multicolumn{2}{|c|}{$\begin{array}{c}\text { Elapsed time in } \\
\text { watermarking embedding } \\
\text { process (seconds) }\end{array}$} & \multirow[t]{2}{*}{ Speedup } & \multirow{2}{*}{$\begin{array}{l}\text { Output file } \\
\text { (watermarked ultrasound } \\
\text { medical images) }\end{array}$} \\
\hline & & Sequential & Parallel & & \\
\hline Ultraound_Sample_1 & 30 & 738.70 & 38.30 & 19.29 & Watermarked_US1 \\
\hline Ultraound_Sample_2 & 15 & 336.40 & 23.80 & 14.13 & Watermarked_US2 \\
\hline Ultraound_Sample_3 & 15 & 332.60 & 22.80 & 14.59 & Watermarked_US3 \\
\hline
\end{tabular}

The elapsed time is actually depending on many factors such as processor type, clock rate, memory speeds and use of memory catches, location of code in memory, compiler efficiency, and compiler optimization technique.

7.2.2. Watermarking Authentication Process. Different to watermarking embedding process, watermarking authentication process is to verify whether there is any tampering that occurred in the watermarked ultrasound medical images and then recovered the tampered frame to its original state. In Table 7, three watermarked ultrasound medical images have been fully tampered and the speedup factor ranges within 2.92 3.64. The speedup factor is lesser as compared to watermarking embedding process. This is because, firstly, watermarking authentication algorithm has more if-else branches as compared to watermarking embedding algorithm and, secondly, watermarking authentication process needs to return two results (a string of tampered frames and concatenated ultrasound frames), whereas watermarking embedding process just returns one result, that is, concatenated frames of watermarked ultrasound medical images.

In watermarking authentication process also is pixel oriented; therefore the different sources have shown a little impact on elapsed time. It could be observed in Figure 11 that watermarking authentication process in sequential mode is proportional to frame size but it is not the case in parallel mode; the elapsed time does not have many changes in parallel mode at different frame size.

7.2.3. Overall Performance of Watermarking Embedding and Authentication Process. The whole package of watermarking process is involving two steps: (1) watermarking embedding process and (2) watermarking authentication process. Therefore it is necessary to test the overall elapsed time involved in both processes. The high speedup in watermarking embedding process is compromised by the low speedup in watermarking authentication process. Table 8 has shown that the overall speedup factor for three ultrasound medical images samples ranges within 5.21 6.60. 
TABLE 7: Sequential versus parallel watermarking authentication process in elapsed time.

\begin{tabular}{|c|c|c|c|c|c|}
\hline \multirow{2}{*}{$\begin{array}{l}\text { Input file (watermarked ultrasound } \\
\text { medical images that have been fully } \\
\text { tampered) }\end{array}$} & \multirow{2}{*}{$\begin{array}{l}\text { Tampered } \\
\text { frame/total } \\
\text { frame }\end{array}$} & \multicolumn{2}{|c|}{$\begin{array}{l}\text { Elapsed time in watermarking } \\
\text { authentication process (seconds) }\end{array}$} & \multirow[t]{2}{*}{ Speedup } & \multirow{2}{*}{$\begin{array}{l}\text { Output file (tampered } \\
\text { ultrasound file recovered as } \\
\text { original ultrasound file) }\end{array}$} \\
\hline & & Sequential & Parallel & & \\
\hline Tampered_Watermarked_US1 & $30 / 30$ & 597.1 & 164.2 & 3.64 & Recovered_US1.dcm \\
\hline Tampered_Watermarked_US2 & $15 / 15$ & 270.0 & 92.6 & 2.92 & Recovered_US2.dcm \\
\hline${ }^{*}$ Tampered_Watermarked_US3_a & $15 / 15$ & 241.2 & 73.3 & 3.29 & Recovered_US3_a.dcm \\
\hline${ }^{*}$ Tampered_Watermarked_US3_b & $15 / 15$ & 259.1 & 71.0 & 3.64 & Recovered_US3_b.dcm \\
\hline
\end{tabular}

*Tampered_Watermarked_US3_a and Tampered_watermarked_US3_b are from the same source but have tampered differently.

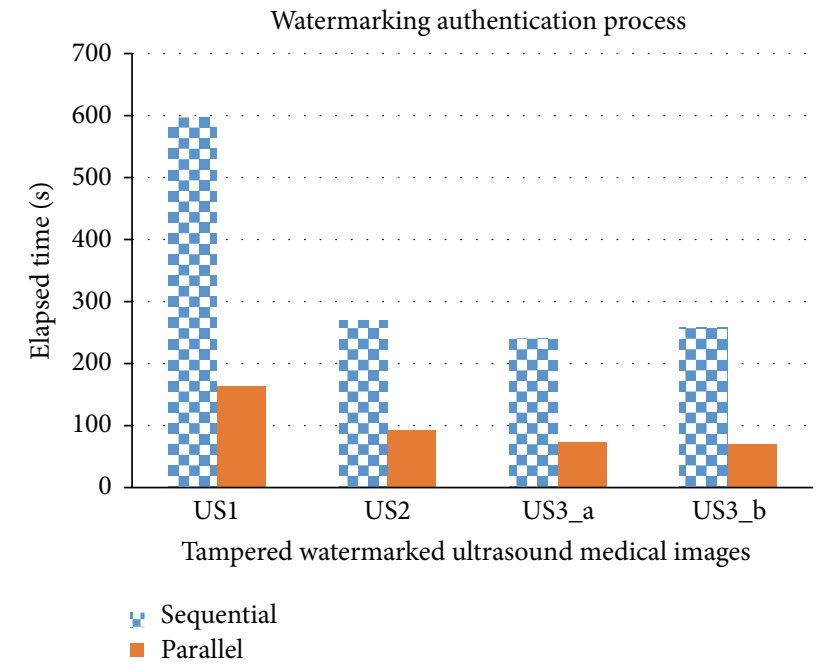

FIGURE 11: Sequential versus parallel watermarking authentication process in elapsed time.

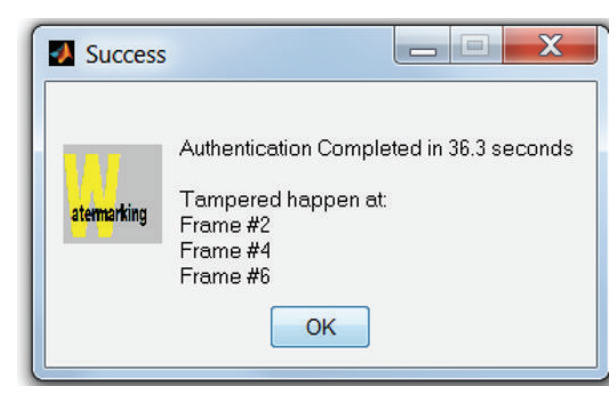

FIGURE 12: A message displayed after watermarking authentication process has been completed.

7.3. Robustness to Tampering. In order to demonstrate the tamper localization function in detecting forgery, counterfeited images were created by manually modifying the pixel values in the watermarked images using image processing software-ImageJ 1.46r. Figure 13 shows an example of tampering on three frames (frames numbers 2, 4, and 6) in ultrasound watermarked medical images.

Different set of tampered watermarked ultrasound medical images has been used to test the effectiveness and efficiency of the tamper detection, localization, and recovery function in multiframes environment (as shown in
TABLE 8: The overall elapsed time comparison between sequential and parallel version of watermarking process (embedding plus authentication).

(a) Overall elapsed time taken in watermarking process on Ultrasound_ Sample_1.dcm

\begin{tabular}{lccc}
\hline Watermarking process & Sequential & Parallel & Speedup \\
\hline Embedding & 738.70 & 38.30 & 19.29 \\
Authentication & 597.10 & 164.20 & 3.64 \\
Overall time taken & 1335.80 & 202.50 & 6.60 \\
\hline
\end{tabular}

(b) Overall elapsed time taken in watermarking process on Ultrasound Sample_2.dcm

\begin{tabular}{lccc}
\hline Watermarking process & Sequential & Parallel & Speedup \\
\hline Embedding & 336.40 & 23.80 & 14.13 \\
Authentication & 270.00 & 92.60 & 2.92 \\
Overall time taken & 606.40 & 116.40 & 5.21 \\
\hline
\end{tabular}

(c) Overall elapsed time taken in watermarking process on Ultrasound Sample_3.dcm

\begin{tabular}{lccc}
\hline Watermarking process & Sequential & Parallel & Speedup \\
\hline Embedding & 332.60 & 22.80 & 14.59 \\
Authentication & 241.2 & 73.3 & 3.29 \\
Overall time taken & 573.8 & 96.1 & 5.97 \\
\hline
\end{tabular}

Table 9). The function's effectiveness was measured by checking whether it could detect the tampered frame number in multiframes environment (as shown in Figures 12 and 13) and is able to recover to its original form (as shown in Figure 14). The function's efficiency was measured by comparing the elapsed time taken for both sequential and parallel watermarking authentication process (as shown in Figure 15). Both effectiveness and efficiency testing were performed while testing the function's robustness to tampering.

For tampered free watermarked ultrasound medical images, the initial setup of parallel authentication process such as determining number of cores and dividing and distributing frames to each core was time consuming; therefore it takes longer processing time than sequential version.

For both sequential and parallel watermarking authentication process, the elapsed time is proportional to the total number of tampered frames. Relative to sequential watermarking authentication, the efficiency of parallel watermarking authentication showed remarkably when there are more tampered frames. In summary, the elapsed time for 

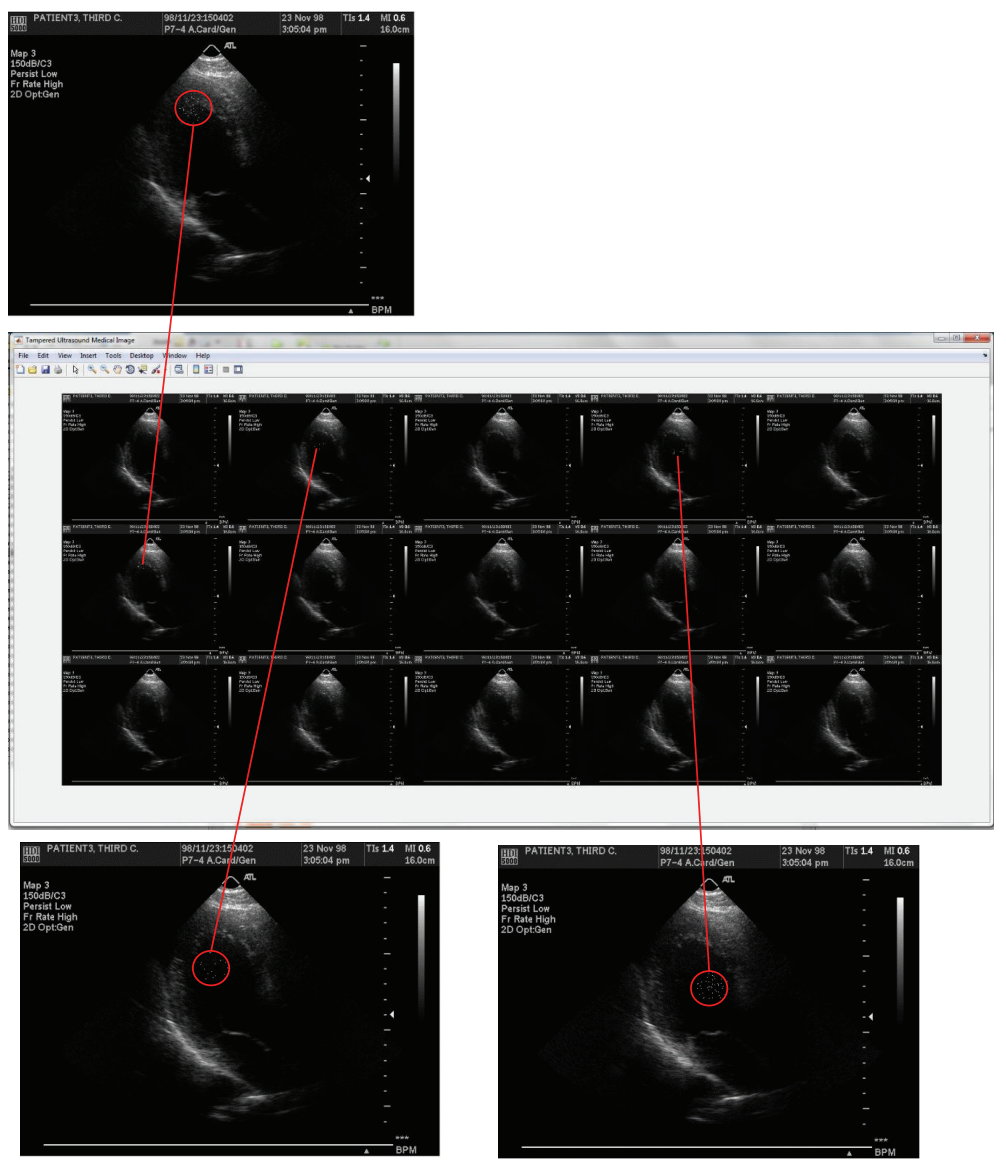

FIGURE 13: Ultrasound medical images tampered on frame numbers 2, 4, and 6.

TABLE 9: A table of comparison on elapsed time of watermarking authentication process on different sets of tampered frames in watermarked ultrasound medical images.

\begin{tabular}{|c|c|c|c|c|c|c|}
\hline \multirow{2}{*}{ Tampered watermarked US images } & \multicolumn{6}{|c|}{ Elapsed time for watermarking authentication process } \\
\hline & Seq & $\mathrm{D}$ & $\mathrm{R}$ & Par & $\mathrm{D}$ & $\mathrm{R}$ \\
\hline Tampered_0_frames & 18.2 & Yes & Yes & 23.1 & Yes & Yes \\
\hline Tampered_3_frames & 56.9 & Yes & Yes & 35.3 & Yes & Yes \\
\hline Tampered_6_frames & 97.0 & Yes & Yes & 39.1 & Yes & Yes \\
\hline Tampered_9_frames & 139.9 & Yes & Yes & 53.8 & Yes & Yes \\
\hline Tampered_12_frames & 241.6 & Yes & Yes & 58.2 & Yes & Yes \\
\hline Tampered_15_frames & 259.1 & Yes & Yes & 71.0 & Yes & Yes \\
\hline
\end{tabular}

Seq: sequential version (seconds).

Par: parallel version (seconds).

D: able to detect and display the tampered frame number?

$\mathrm{R}$ : able to recover to its original form after authentication process?

parallel watermarking authentication process is varied based on the total number of tampered frames in ultrasound medical images.

With $100 \%$ tampered watermarked ultrasound medical images, the efficiency of parallel version can be calculated based on elapsed time, such as $259.1 / 71=3.65$, which means parallel version can perform 3.65 times faster than sequential version. In other words, sequential watermarking authentication has performed a job all by itself, whereas parallel watermarking authentication has delegated a job to four workers/cores, in which parallel version has performed approximately 4 times faster than sequential version. In conclusion, the speedup factor was approximate to the number of cores since there is little communication between subtasks.

The frame order of tampered frame also is a factor that affects the performance in parallel process (as shown in Figure 16). The black rectangle boxes with bold font represent a tampered frame. Both (a) and (b) have distributed 15 frames 


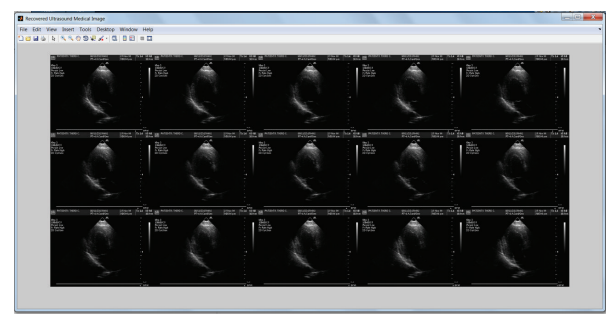

FIGURE 14: Recovered ultrasound medical images after watermarking authentication process.

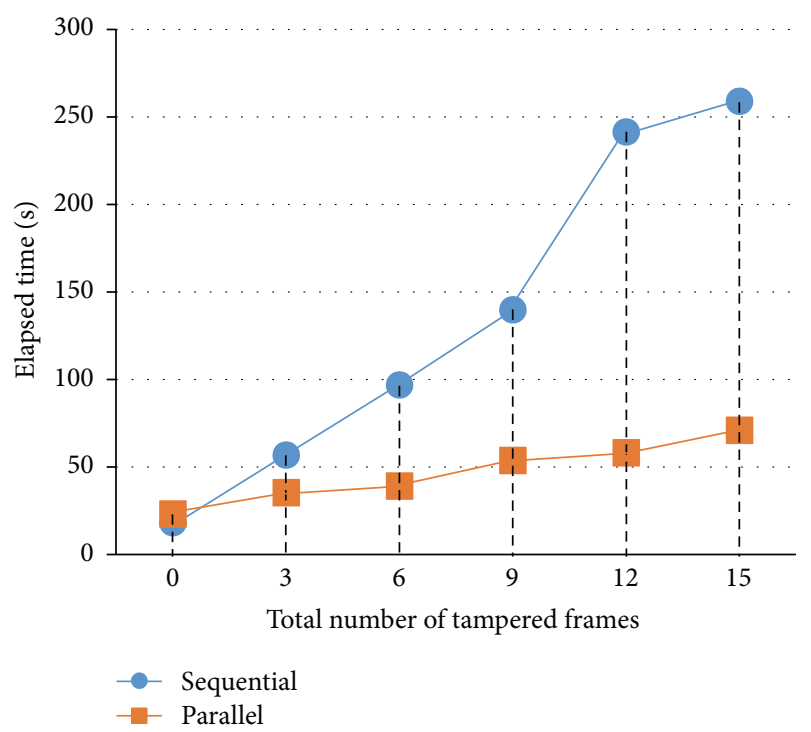

FIGURE 15: Comparison of elapsed time between sequential and parallel watermarking authentication process on different number of tampered frames.

(containing 9 tampered frames) of watermarked ultrasound medical images to 4 cores, respectively. The main difference between (a) and (b) was that the tampered frames were organized in different order. The elapsed time for (a) is 5 seconds lesser than (b). It is because (a) has an even distribution of tampered frames and hence fairer workloads among 4 cores as compared to (b).

\section{Conclusion and Future Work}

The approach used to overcome the performance constraint in sequential watermarking process is by distributing ultrasound frames over multiple cores. The performance constraint for sequential watermarking process can be categorized into two problems: capacity and capability. The capacity problem occurred when the existing hardware and software are unable to perform the anticipated computations in an estimated time [18]. For example, it may not be feasible to conduct watermarking process on a large data size of DICOM files in any reasonable manner. Therefore, to run the entire computation may become impractical even though the existing hardware and software are capable of performing the watermarking process as required. The actual physical

\begin{tabular}{l|l|l|l|l|}
\cline { 3 - 5 } Core 1 & Frame \#1 & Frame \#2 & Frame \#3 & Frame \#4 \\
\cline { 2 - 5 } Core 2 & Frame \#5 & Frame \#6 & Frame \#7 & Frame \#8 \\
\cline { 2 - 5 } Core 3 & Frame \#9 & Frame \#10 & Frame \#11 & Frame \#12 \\
\cline { 2 - 6 } Core 4 4 Frame \#13 & Frame \#14 & Frame \#15 & \\
\cline { 2 - 5 } & & &
\end{tabular}

(a) Elapsed time: 53.8 seconds

\begin{tabular}{l|l|l|l|l|}
\cline { 3 - 5 } Core 1 & Frame \#1 & Frame \#2 & Frame \#3 & Frame \#4 \\
\cline { 2 - 5 } Core 2 & Frame \#5 & Frame \#6 & Frame \#7 & Frame \#8 \\
\cline { 2 - 5 } Core 3 & Frame \#9 & Frame \#10 & Frame \#11 & Frame \#12 \\
\cline { 2 - 5 } Core 4 & Frame \#13 & Frame \#14 & Frame \#15 & \\
\cline { 2 - 6 } & & &
\end{tabular}

(b) Elapsed time: 58.9 seconds

FIGURE 16: The elapsed time taken in different order of tampered frames organization during parallel watermarking authentication process.

constraint, such as the processor speeds or total memory on a system, may cause the problem of capability, in which it will restrict the amount of watermarking processes performed. System upgrades may be a solution for the problem concern, but it is bounded by technology and cost constraint. In this case, the sequential watermarking problem may be partitioned into smaller and manageable parts that can be performed in parallel. Parallel computing with MATLAB is the simplest approach to leveraging multicores processor. However, the maximum number of parallel threads cannot exceed the number of cores available on the system. The performance gain obtained by using multiple cores on a single system is also limited and varies depending on the specific computation and the data size [9]. The proposed parallel watermarking scheme required little effort to separate the ultrasound medical images into a number of parallel tasks due to the little dependency (or communication) between those parallel tasks and achieved a speedup factor that is almost equivalent to the number of cores; for example, quad cores microprocessor will result in a speedup factor of approximately four, which means parallel watermarking process is approximately four times faster than sequential mode in quad cores microprocessor. Hence it could be classified as "embarrassingly parallel problems," a phrase that comments on the ease of parallelizing such applications and the fact that it would be embarrassing for the programmer or compiler to not take advantage of such an obvious opportunity to improve performance [19]. The performance of proposed parallel digital watermarking scheme could be further improved by using graphics processing unit (GPU). Both CPU and GPU could run thousands of threads concurrently, but GPU will have a better performance than CPU due to its larger number of cores possessed relative to CPU; the difference in theoretical performance can differ by a factor ten in favour of the GPU; therefore a parallel digital watermarking operation on GPU is recommended. In many cases a hybrid CPU-GPU implementation yields the best performance. A good example is image registration algorithms, where the GPU can be used to calculate a chosen similarity measure in parallel, while the $\mathrm{CPU}$ can run a serial optimization algorithm. So et al. made a comparison between CPUs and GPUs for ultrasound systems, in terms of power efficiency and cost effectiveness. The conclusion was that a hybrid CPU-GPU system performed 
best [20]. The adoption of either GPU or hybrid CPU-GPU is largely dependent on the parallel adaption in an algorithm; an algorithm that exhibited "embarrassingly parallel problem" will be suitably used in GPU whereas hybrid CPU-GPU is suitably applied in an algorithm that exhibited "fine-grained parallelism." For future work, the proposed method could be applied on Magnetic Resonance Images (MRI) where the ROI could be classified by using weighted-type fractional Fourier transform approach [21] prior to watermarking process. Since the watermarking is pixel oriented, thus it could be also applied on nature images with lesser restriction on image fidelity requirement as compared to medical images.

\section{Conflict of Interests}

The authors declare that there is no conflict of interests regarding the publication of this paper.

\section{References}

[1] C. K. Tan, J. C. Ng, X. Xu, C. L. Poh, Y. L. Guan, and K. Sheah, "Security protection of DICOM medical images using duallayer reversible watermarking with tamper detection capability," Journal of Digital Imaging, vol. 24, no. 3, pp. 528-540, 2011.

[2] Q. Li and N. Memon, "Security models of digital watermarking," in Multimedia Content Analysis and Mining: International Workshop, MCAM 2007, Weihai, China, June 30-July 1, 2007. Proceedings, vol. 4577 of Lecture Notes in Computer Science, pp. 60-64, Springer, Berlin, Germany, 2007.

[3] G. Wang and N.-N. Rao, "A fragile watermarking scheme for medical image," in Proceedings of the 27th Annual International Conference of the Engineering in Medicine and Biology Society (IEEE-EMBS '05), pp. 3406-3409, IEEE, Shanghai, China, January 2005.

[4] S.-C. Liew and J. M. Zain, "The usage of block average intensity in tamper localization for image watermarking," in Proceedings of the 4th International Congress on Image and Signal Processing (CISP '11), pp. 1044-1048, Shanghai, China, October 2011.

[5] S.-C. Liew, S.-W. Liew, and J. M. Zain, "Tamper localization and lossless recovery watermarking scheme with ROI segmentation and multilevel authentication," Journal of Digital Imaging, vol. 26, no. 2, pp. 316-325, 2013.

[6] S.-C. Liew and J. M. Zain, "Reversible medical image watermarking for tamper detection and recovery," in Proceedings of the 3rd IEEE International Conference on Computer Science and Information Technology (ICCSIT '10), pp. 417-420, Chengdu, China, July 2010.

[7] J. M. Zain and A. R. M. Fauzi, "Medical image watermarking with tamper detection and recovery," in Proceedings of the 28th Annual International Conference of the IEEE Engineering in Medicine and Biology Society (EMBS '06), pp. 3270-3273, New York, NY, USA, September 2006.

[8] B. W. R. Agung and P. F. P. Adiwijaya, "Medical image watermarking with tamper detection and recovery using reversible watermarking with LSB modification and run length encoding (RLE) compression," in Proceedings of the IEEE International Conference on Communication, Networks and Satellite (ComNetSat '12), pp. 167-171, Bali, Indonesia, July 2012.

[9] S. Samsi, V. Gadepally, and A. Krishnamurthy, "MATLAB for signal processing on multiprocessors and multicores," IEEE Signal Processing Magazine, vol. 27, no. 2, pp. 40-49, 2010.
[10] S. P. Wang and R. S. Ledley, "Advanced computer architecture," in Computer Architecture and Security Fundamentals of Designing Secure Computer Systems, John Wiley \& Sons, New York, NY, USA, 2013.

[11] J. Degroote and J. Vierendeels, "Multi-solver algorithms for the partitioned simulation of fluid-structure interaction," Computer Methods in Applied Mechanics and Engineering, vol. 200, no. 2528, pp. 2195-2210, 2011.

[12] R. Cieszewski, M. Linczuk, K. Pozniak, and R. Romaniuk, "Review of parallel computing methods and tools for FPGA technology," in Photonics Applications in Astronomy, Communications, Industry, and High-Energy Physics Experiments 2013, vol. 8903 of Proceedings of SPIE, Wilga, Poland, October 2013.

[13] F. P. Brooks, The Mythical Man-Month: Essays on Software Engineering, Addison-Wesley, Reading, Mass, USA, 1996.

[14] P. Vicat-Blanc, S. Soudan, R. Guillier, and B. Goglin, "Utilization of network computing technologies," in Computing Networks: From Cluster to Cloud Computing, chapter 2, John Wiley \& Sons, Hoboken, NJ, USA, 2013.

[15] The MathWorks, How Parallel Computing Products Run a Job, 2015, http://www.mathworks.com/help/distcomp/howparallel-computing-products-run-a-job.html.

[16] I. J. Cox, M. L. Miller, and J. A. Bloom, Digital Watermarking, Morgan Kaufmann, San Francisco, Calif, USA, 2002.

[17] Y.-D. Zhang, S.-H. Wang, X.-J. Yang et al., "Pathological brain detection in MRI scanning by wavelet packet Tsallis entropy and fuzzy support vector machine," SpringerPlus, vol. 4, article 716, 2015.

[18] B. V. Krishna and K. Baskaran, "Parallel computing for efficient time-frequency feature extraction of power quality disturbances," IET Signal Processing, vol. 7, no. 4, pp. 312-326, 2013.

[19] A. Leykin, J. Verschelde, and Y. Zhuang, "Parallel homotopy algorithms to solve polynomial systems," in Mathematical Software-ICMS 2006: Second International Congress on Mathematical Software, Castro Urdiales, Spain, September 1-3, 2006. Proceedings, vol. 4151 of Lecture Notes in Computer Science, pp. 225-234, Springer, Berlin, Germany, 2006.

[20] H. K.-H. So, J. Chen, B. Y. S. Yiu, and A. C. H. Yu, "Medical ultrasound imaging: to GPU or not to GPU?" IEEE Micro, vol. 31, no. 5, pp. 54-65, 2011.

[21] Y.-D. Zhang, S. Chen, S.-H. Wang, J.-F. Yang, and P. Phillips, "Magnetic resonance brain image classification based on weighted-type fractional Fourier transform and nonparallel support vector machine," International Journal of Imaging Systems and Technology, vol. 25, no. 4, pp. 317-327, 2015.

[22] R. A. Jain and D. V. Padole, "Scalable and flexible heterogeneous multi-core system," International Journal of Advanced Computer Science and Applications, vol. 3, no. 12, pp. 174-179, 2012.

[23] G. Luo and D. Zhang, "Efficiency improvement for dataprocessing of partial discharge signals using parallel computing," in Proceedings of the 10th IEEE International Conference on Solid Dielectrics (ICSD '10), pp. 1-4, IEEE, Potsdam, Germany, July 2010. 


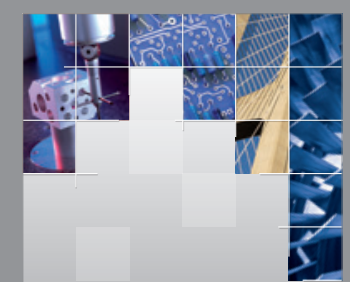

\section{Enfincering}
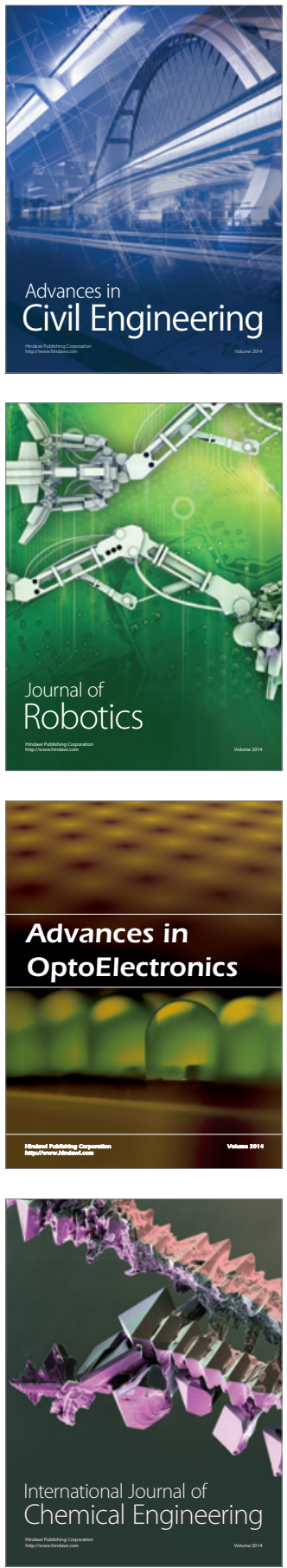

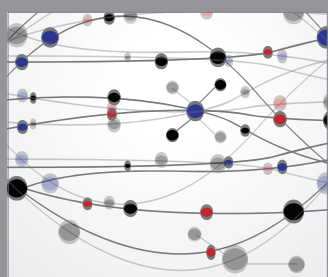

The Scientific World Journal

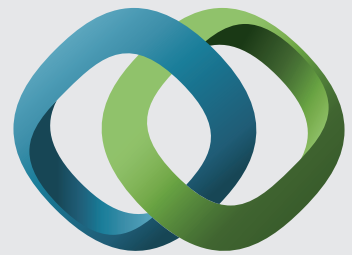

\section{Hindawi}

Submit your manuscripts at

http://www.hindawi.com
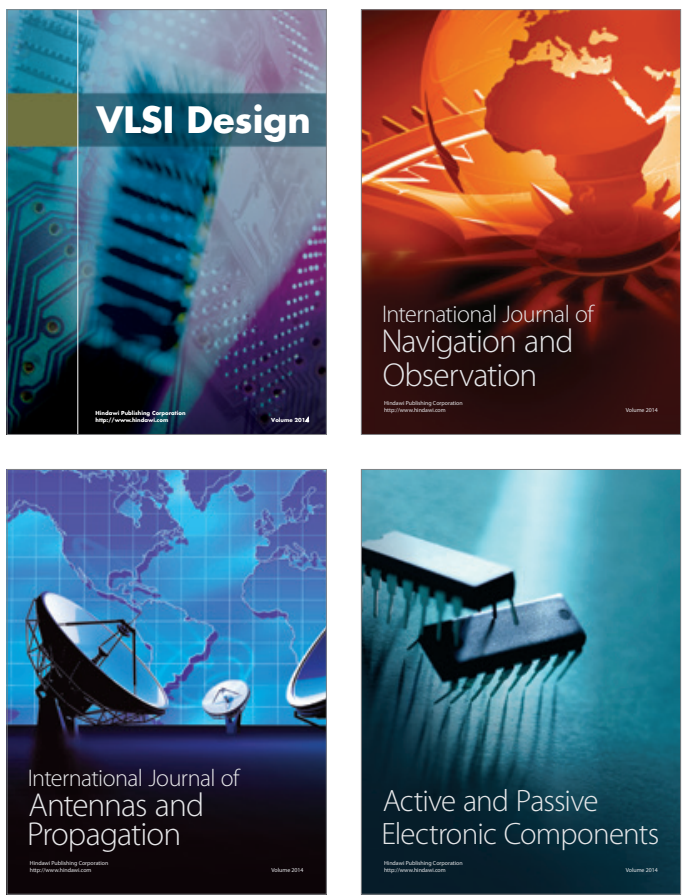
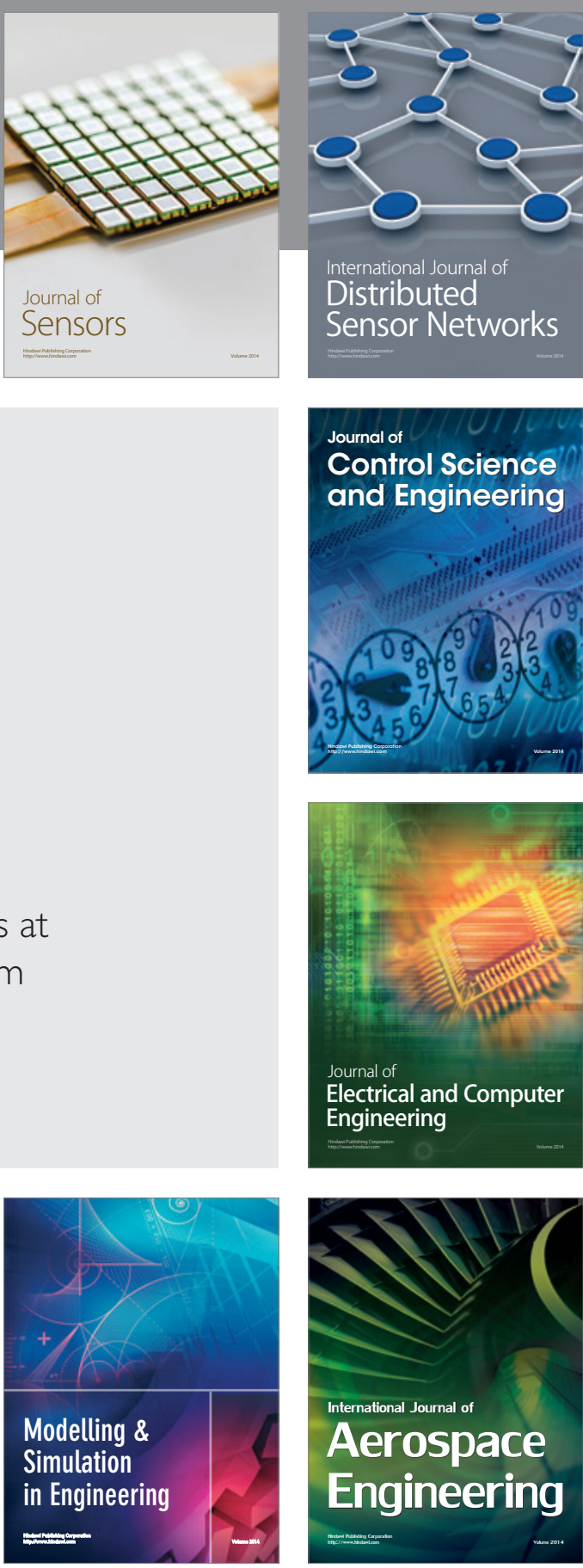

International Journal of

Distributed

Sensor Networks

Journal of

Control Science

and Engineering
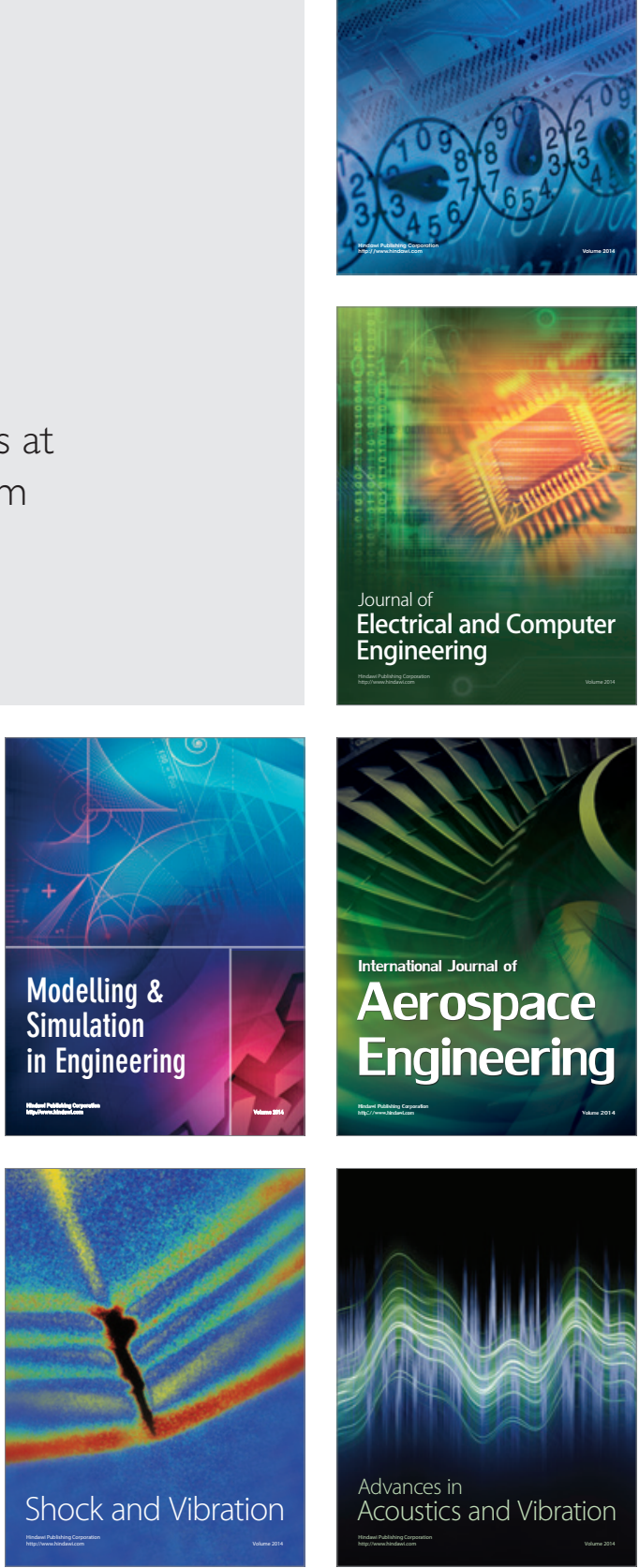